\title{
Timescale of variation and the size of the accretion disc in active galactic nuclei
}

\begin{abstract}
M. R. S. Hawkins
Institute for Astronomy (IfA), University of Edinburgh, Royal Observatory, Blackford Hill, Edinburgh EH9 3HJ, UK

e-mail: mrsh@roe.ac.uk

Received 22 August 2006 / Accepted 2 November 2006

ABSTRACT

This paper sets out to measure the timescale of quasar variability with a view to new understanding of the size of accretion discs in active galactic nuclei. Previous attempts to measure such timescales have been based on sparsely sampled data covering small ranges of time. Here we combine data from two large scale monitoring programmes to obtain Fourier power spectra of light curves covering nearly three orders of magnitude in frequency in blue and red passbands. If the variations are interpreted as due to gravitational microlensing, then timescale measurements in the observer's frame imply a minimum mass for the microlensing bodies of around $0.4 M_{\odot}$. On the assumption that the variations are intrinsic to the quasars, a correction must be made for time dilation. In this case the power spectrum shows a break corresponding to a timescale of about 11 years. This timescale is used to measure the size of the accretion disc, which is found to be about $10^{-2}$ pc or 10 light days, in agreement with limits set by self-gravitation and coincident with the broad line region of the active galactic nucleus. It is suggested that the broad line region may be associated with the break up of the outer part of the accretion disc.
\end{abstract}

Key words. quasars: general - galaxies: active

\section{Introduction}

When quasars were first discovered in the 1960s a fundamental property which defined their nature was the substantial variation in brightness in optical passbands, observed over a timescale of a few months. This put severe constraints on the maximum size of the emitting region, and was largely responsible for the eventual understanding of the overall structure of an active galactic nucleus (AGN). Since these early observations, much effort has been put into establishing the minimum timescale on which AGN can be seen to vary in brightness, both in optical and X-ray passbands (Klimek et al. 200; Stalin et al. 2004). More problematic has been the attempt to determine the longest timescale on which AGN show significant variations. Over the last 20 years or so a number of groups have published the results of monitoring programmes covering a decade or more (Hawkins 2002; Peterson et al. 1999; Cristiani et al. 1996; Hook et al. 1994; Trevese et al. 1994). Most of these programmes have been aimed at establishing correlations between a measure of the degree of variability, typically amplitude, and parameters such as luminosity or redshift. Although there is some divergence in the results, there now seems reasonable agreement that there is an anti-correlation between luminosity and amplitude in the sense that more luminous quasars vary with smaller amplitudes, but there is no convincing detection of a correlation between amplitude and redshift. These studies do however suffer from several drawbacks, including the lack of a satisfactory definition of amplitude which is independent of the timespan of the observations, and the degeneracy between redshift and luminosity which are correlated in most quasar samples.

A new approach to the analysis of AGN variability has recently become possible with the publication of a very large quasar sample from the Sloan Digital Sky Survey (SDSS)
(Schneider et al. 2003). Several groups have used this data to analyse quasar variability, but in particular two groups (Sesar et al. 2006; de Vries et al. 2005) have combined this data with measures of early epoch material from the Palomar Sky Surveys to give up to three well separated epochs. They have then taken advantage of an assumed time dilation effect to recover rest frame light curves which result in a wide range of time differences, and enable them to construct a well sampled structure function. This neat idea seems to work moderately well to give the rough shape of the underlying structure function, but there are many systematic effects which limit the amount of information which can be extracted. These include the challenge of correcting for the different passbands in which the three surveys were taken, large and hard to quantify Malmquist biases and the differing effect of the underlying host galaxy in the different media of the three surveys.

An important parameter to be obtained from a monitoring programme is the characteristic timescale of variation. In those surveys where a structure function or Fourier power spectrum has been constructed from the light curves it has been generally found that there is a power law rise towards longer timescales, but the question of where this power law relation turns over has not so far been established in a convincing way. This break point which gives the characteristic timescale of variation is of great importance in establishing the overall size of the accretion disc, and can also constrain the mass transfer rate. In the event that variations are caused by microlensing (Hawkins 1996), it is a measure of the mass of the microlensing bodies. The early values for timescale of variation from this monitoring programme (Hawkins 1996) were based firstly on visual estimations of a representative value from examination of the light curves. A second approach involved an attempt to model the observed autocorrelation function with simulated light curves from the literature. 
Neither of these approaches can be considered as anything more than lower limits, especially in view of the relatively short run of data available at that time. The problem of timescale measurement was revisited some years later (Hawkins 2001) when Fourier power spectrum analysis was used, along the lines of the present paper. In this case an unbroken power law was observed, implying that no characteristic timescale was measureable with the available data.

A considerable amount of work has already been undertaken to measure cut-off frequencies for AGN variation in X-ray bands (Uttley et al. 2002; Edelson \& Nandra 1999). There now seems little doubt that characteristic timescales have been measured, which are typically about one month, with an index for the power law of around -1.8 , and the position of the break is interpreted as a measure of the black hole mass (Uttley et al. 2002).

In this paper we assemble the best available optical data from timescales of a few days to thirty years. We then investigate methods for analysing the data with a view to measuring timescales of variation both in the observer's frame and quasar rest frame. From the combined data we then construct Fourier power spectra spanning three orders of magnitude in time with a view to measuring timescale of variation. Finally, we review the implications of these results in the context of various models for the observed optical variations.

\section{The quasar monitoring programme}

\subsection{Photometric observations}

This investigation into the timescale of variability of AGN is primarily based on a 28 year monitoring programme with photographic plates taken with the UK $1.2 \mathrm{~m}$ Schmidt telescope at Siding Spring Observatory in Australia. The survey area comprises the central unvignetted part of ESO/SERC field 287 centred on $21^{\mathrm{h}} 28^{\mathrm{m}},-45^{\circ}(1950)$ and covers approximately 20 square degrees. Over 300 plates were taken of this field during the lifetime of the UK Schmidt, in a variety of passbands from $U$ to $I$, as well as objective prism and other more specialised plates, but for the purposes of the present project we restrict our attention to a series of plates taken every year for 26 years in the $B_{J}$ (IIIa-J/GG395) passband from 1977 to 2002, and for 23 years in the $R$ (IIIa-F/RG630) passband from 1980 to 2002. In most years 4 plates were obtained, and details of all but the last year are given by Hawkins (2003).

The plates were measured by the COSMOS and SuperCOSMOS machines at the Royal Observatory Edinburgh, which produced a catalogue of approximately 200000 objects to a completeness limit of $B_{J}=21.5$. There was also a period of 15 years from 1983 to 1997 where there was a homogeneous run of data comprising 4 good $B_{J}$ plates every year, and these were digitally stacked to provide a second dataset with a completeness of about $B_{J}=22$. The plates were calibrated using deep CCD sequences (Hawkins 1996), and reduced to the same zero-point using local photometric transformations. The photometric accuracy on each measurement was obtained from repeat measures of the same objects on several different plates, and found to be $\pm 0.15 \mathrm{mag}$ in the magnitude range appropriate to the quasar sample. The overall rms variation on the light curves, including mean magnitudes where more than one plate per year was available was $\pm 0.11 \mathrm{mag}$. This was evaluated directly by measuring the root mean square ( $\mathrm{rms}$ ) variation of the light curves of samples of stars of similar colour and apparent magnitude distribution, assumed to be non-variable.

\subsection{Sample selection}

The sample of candidate quasars or AGN for spectroscopic confirmation were selected by a number of methods. These included ultraviolet excess, typically with a limit of $B_{J}-U<0.0$, and variability with an amplitude over 28 years of $\delta B_{J}>0.35$. The exact criterion for variability is given in Hawkins (1996). In addition to these basic selection criteria, other methods were used including objective prism searches, and candidates from radio surveys with the Molongolo and Australia telescopes, but in practice all such candidates were already selected by other methods. Variability has proved to be a particularly powerful way of detecting quasars, and it has been shown (Hawkins 2000) that nearly all quasars can be detected on the basis of variability after 15 years of survey data.

Separate sampling programmes were carried out with the stacked and single plate data. Although there was an approximate $75 \%$ overlap between the two selection lists, the stacked data contained fainter objects below the limit of the single plate data, whereas the single plate data had less of an overcrowding problem and contained objects which were merged in the stacked data. In all there were about 2000 good candidates suitable for spectroscopic observations to establish whether they were AGN, and if so to measure their redshifts.

\subsection{Spectroscopy}

Spectroscopic follow up of candidates from the monitoring programme was commenced some 20 years ago, and as the wide field capability of spectrographs has improved the number of confirmed AGN has rapidly increased. The first sample to be analysed (Hawkins 1996) comprised some 400 quasars with redshifts. This had increased to around 600 for the analysis by Hawkins (2002), as a result of a number of small time allocations with single slit spectrographs. The most recent and largest award of time was in July 2002 with the $2 \mathrm{dF}$ multi-object spectrograph on the Anglo-Australian Telescope. This resulted in a homogeneous set of redshifts for about $1000 \mathrm{AGN}$, including a number of re-observations, and brought the total number of confirmed AGN with redshifts to about 1500 in the 20 square degrees of the survey. The redshifts were measured to an accuracy of approximately $\pm 0.2 \%$, evaluated from multiple observations of the same object; more details of the procedures used are given by Hawkins (2004).

The above procedure resulted in a sample of 814 quasars for which light curves in the $B_{J}$ passband covering 26 yearly epochs were measured. This included the requirement that there should be no missing epochs. The redshifts of the quasars in the sample range from 0.15 to 3.8 , and the luminosities from $M_{B}=-20$ to $M_{B}=-28$. The distributions of these two quantities are shown in the form of histograms in Fig. 1. Light curves in $R$ were also available for the quasar sample, but the restriction that there should be no missing epochs, combined with the blue colour of most quasars, meant that the sample of acceptable red passband light curves was reduced to 403. These two sets of light curves formed the basis for the timescale analysis described below.

\subsection{The MACHO quasars}

In earlier work on the sample of quasars in field 287 described above, a major limitation to the measurement of timescales has been the length of the baseline in the $\log / \log$ plot (e.g. Hawkins 2001, 2002). To date the approach has been to extend as far as 
Quasars

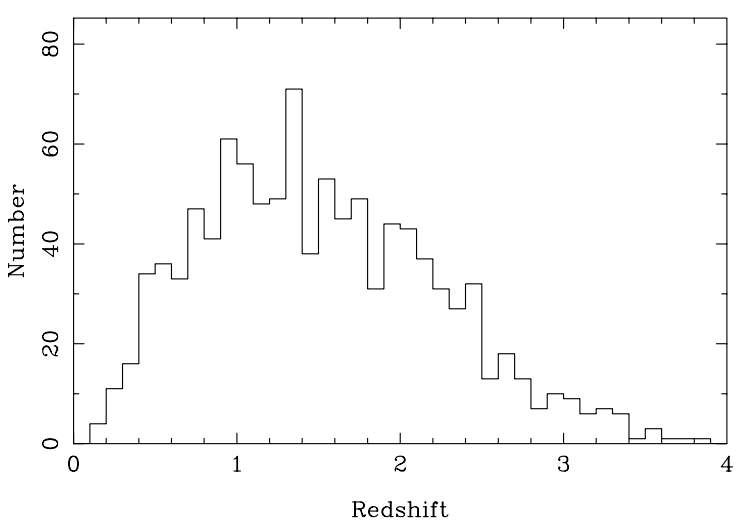

Quasars

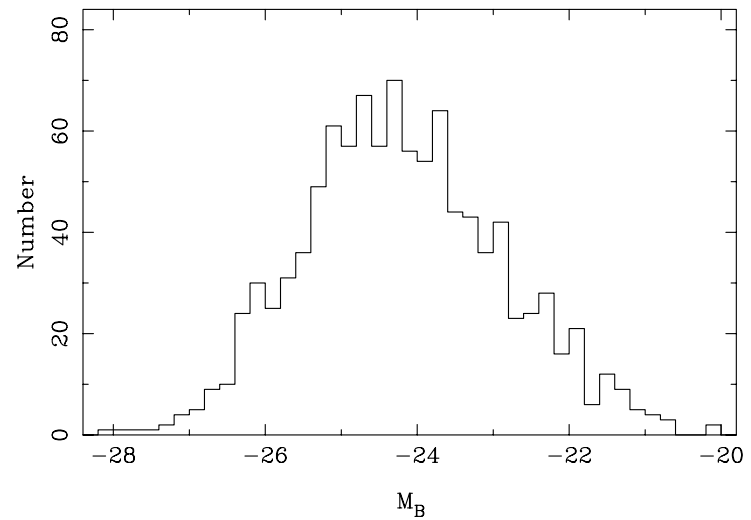

Fig. 1. Histograms of the redshift (left hand panel) and absolute magnitude (right hand panel) of quasars in the monitoring survey.

possible the length of the time baseline, but the logarithmic nature of the increase makes this a strategy of diminishing returns. One way of improving the chances of detecting a turnover is to provide a longer baseline by including observations taken over shorter timescales. An ideal database for this purpose is the long set of observations taken for the MACHO project. The idea of this well-known project was to monitor several million stars in the direction of the Magellanic Clouds with a typical frequency of a few days to look for the characteristic signature of a Galactic microlensing event (Alcock et al. 1997).

Although the overwhelming majority of the objects in the MACHO monitoring programme were stars, there were inevitably a number of quasars in the area of interest which were also observed. These have recently been collated and published with their redshifts (Geha et al. 2003), and form a very useful, if rather small, database for measuring short timescale variations down to a few days. There is a total of 59 quasars in their database, including their new discoveries and those already known. The redshifts and absolute magnitudes of these quasars lie in the ranges $0.1<z<2.8$ and $-28<M_{B}<-20$, and follow similar distributions to those shown in Fig. 1.

The observations were made with a CCD camera in $B$ and $R$ bands on every night permitted by the weather and other logistical constraints. Occasionaly more than one observation per night was obtained. The photometric accuracy is recorded for each observation, and is quite variable depending on seeing conditions, level of contamination by neighbouring objects and the brightness of the object. A typical error estimate on a single measurement is $\pm 0.1 \mathrm{mag}$. For the purposes of the work in this paper, the observations were put into bins of 10 or 50 days, and the errors combined in quadrature. For the 50 day bins this resulted in a typical error per epoch of about $\pm 0.03 \mathrm{mag}$.

Although the full set of MACHO observations covers more than 7 years, the patchiness of the coverage for some quasars combined with the requirement for uninterrupted sampling meant that for the 50 day bins the original total of 59 quasars was reduced in the blue passband to 48 , with a continuous run of 51 epochs, and in the red to 35 over 36 epochs. For the 10 day bins useful data was only obtained in the periods of relatively intense observations due to the requirement that there should be a continuous run of data. This meant that the total was reduced to 32 quasars in the blue passband and 25 in the red, both measured over 33 epochs. These were the light curves used for the analysis described below.

\section{Time series analysis}

It has been known for some time (Cristiani et al. 1996; Hawkins 1996) that the spectrum of variations of AGN, when measured over several years, increases in power towards longer timescales. The Fourier power spectrum of the variations has been shown to be close to a power law in form (Hawkins 2001), but the question of where this power law turns over at long timescales, and hence the identification of a characteristic timescale, has remained open. For example, Hawkins (2001) concluded that the Fourier power spectra of quasar light curves were adequately fitted by a power law relationship with no turnover, implying that the run of data was still too short to reach any characteristic timescale. In another paper (Hawkins 2002), structure functions were used to match the presentation of simulated data, but due to windowing effects producing apparently spurious features it was only possible to compare slopes, and not any more detailed features.

It is clear that the measurement of timescales in light curves requires a statistical treatment of the data which enables the detection of a break or other feature in the spectrum of variations, combined with a sufficiently long run of photometric measurements. To date, two functional forms have principally been used for the analysis of timescales, each of which has points in favour and against. We discuss and illustrate these in the following two subsections.

\subsection{The structure function}

The most frequently used functional form for the analysis of quasar variations is undoubtedly the structure function (Vanden Berk et al. 2004; de Vries et al. 2003; Hawkins 2002; Cristiani et al. 1996; Trevese et al. 1994), together with the closely related auto-correlation function (Hawkins 1996). In addition, theoretical models of quasar variations have been published in the form of structure functions (Kawaguchi et al. 1998; Hawkins 2002). The structure function $S(\tau)$ is usually defined as $S(\tau)=\sqrt{\frac{1}{N(\tau)} \sum_{i<j}\left[m\left(t_{j}\right)-m\left(t_{i}\right)\right]^{2}}$

where $m\left(t_{i}\right)$ is the magnitude measure at epoch $t_{i}$, and the sum runs over the $N(\tau)$ epochs for which $t_{j}-t_{i}=\tau$, and only includes those time differences where data is available. If the structure function is for more than one object, then the summation runs over time intervals for all the light curves in the sample. This 

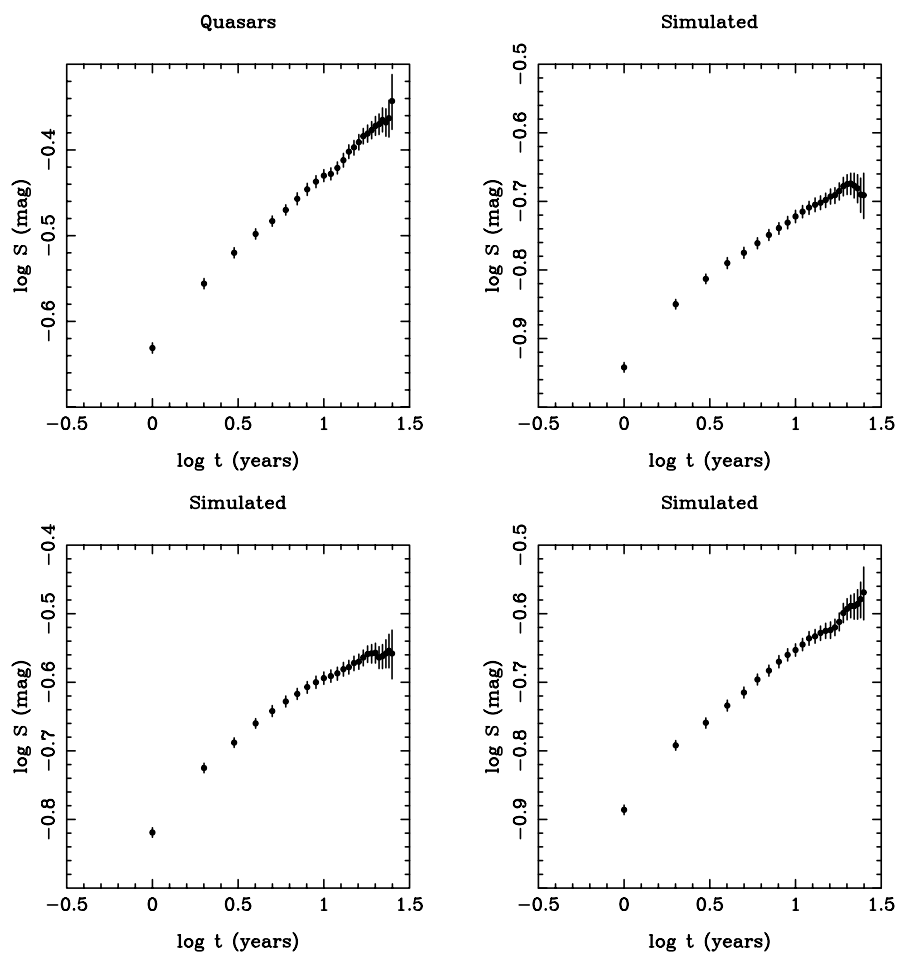

Fig. 2. Structure functions for the sample of 814 quasars in Field 287 (top left hand panel) and for three realisations of 814 simulated light curves with a similar power spectrum of variations and the same sampling.

results in one of the most important advantages of the structure function over other methods, that is its insensitivity to gaps in the run of data, especially on short timescales. This has proved of great benefit to surveys which rely on archival observations taken at irregular intervals for their data.

To set against their usefulness for analysing unevenly sampled data sets, there are some serious problems associated with structure functions. Perhaps the best known is the difficulty of error analysis, associated with the correlation of errors from point to point. This has the effect of producing what at first sight appear to be real features in the form of changes of slope or breaks, and makes the detailed interpretation of the shape of a structure function very difficult. To illustrate the problem, we show in the top left hand panel of Fig. 2 the structure function for the sample of quasars in Field 287. The plot is constructed by calculating the mean structure function for the sample of 814 blue passband light curves, as described above. In the $\log / \log$ plot, the structure function increases to longer timescales in a roughly linear mannner, indicating a power law relationship. There is no sign of a significant turn over or break, although there is the possibility of features associated with the wavy departures from a strictly linear increase.

In order to investigate the significance of the shape of the quasar structure function, simulated light curves were constructed with known statistics of variation. Each simulation was made by defining a pure power law power spectrum for a large number of data points, taking the square root, applying a random phase and taking the inverse Fourier transform. The index of the power law was -1.2 , chosen to give a structure function slope close to that measured for the quasars. This produced a long light curve with the required variability characteristics. Out of this were cut 814 individual light curves of the same length (26 epochs) as for the observations described in Sect. 2.
Structure functions were then calculated for the sets of light curves as described above, three of which are shown as $\log / \log$ plots in the remaining panels of Fig. 2.

It will be seen that for short timescales the simulated structure functions increase with time in a roughly linear fashion in all three cases. However, beyond about 5 years the three functions diverge, with one eventually turning over at longer timescales while the other two continue to rise at different rates, with some fluctuations. If the only structure function available were the one in the top right hand panel, a naive interpretation would suggest a power law relationship with a long timescale cut-off. This interpretation is made more plausible by predictions in the literature for such behaviour in AGN structure functions for a wide range of models (Hawkins 2002; Kawaguchi et al. 1998). There is also the possibility of other features associated with the wavy departures from a strictly linear increase. In fact, it is clear that from the same power law spectrum, a variety of structure function shapes can result. The data in the top left panel of Fig. 2 is largely the same as that used in Hawkins (2002), and has a very similar shape. It illuminates the point made in that paper that the only reason for working with structure functions is that the simulations were published in that form.

It is easy to show that for an infinite run of observations, the structure function for a power law spectrum of variations is also a power law. The simulated structure functions in Fig. 2 clearly depart from power law shapes, especially towards long timescales. This is due to the unpredictable effect of windowing on a finite run of data, which produces different morphologies for different sets of light curves, despite their being drawn from the same underlying spectrum of variations.

The data in Fig. 2 make clear the unsuitability of structure functions for the analysis of short runs of data, and especially for the identification of cut-offs to be used for the measurement of timescale. It is of course true that because of irregular sampling in many sets of data, it is the only feasible choice. In this case it is probably best to confine the analysis to an average measurement of the power law slope which is less affected by windowing effects, although the presence of noise in the light curves will alter the observed index.

\subsection{The Fourier power spectrum}

For an infinite run of data the Fourier power spectrum and structure function or auto-correlation function are essentially equivalent, one being the Fourier transform of the other. However, for finite datasets, and especially for the short datasets typically encountered in AGN monitoring programmes, the windowing function has a significant and very different effect in the two cases. We define the Fourier power spectrum $P(s)$ as

$P\left(s_{i}\right)=\frac{\tau}{N}\left(\sum_{j=1, N} m\left(t_{j}\right) \cos \frac{2 \pi i j}{N}\right)^{2}+\frac{\tau}{N}\left(\sum_{j=1, N} m\left(t_{j}\right) \sin \frac{2 \pi i j}{N}\right)^{2}$

where $i$ runs over the $N$ equally spaced epochs of observation separated by time $\tau$, and $m\left(t_{j}\right)$ is the magnitude at epoch $t_{j}$. In the case of a sample of light curves, the integration for each frequency continues over all sample members.

The main advantage of the structure function, its insensitivity to irregular sampling, is not shared by Fourier transforms. Here, unevenly sampled data leads to aliassing in the form of spurious features which can cause great difficulties in the measurement and interpretation of power spectra. However, given evenly spaced observations, errors are straightforward to calculate as each Fourier frequency is essentially independent, and 

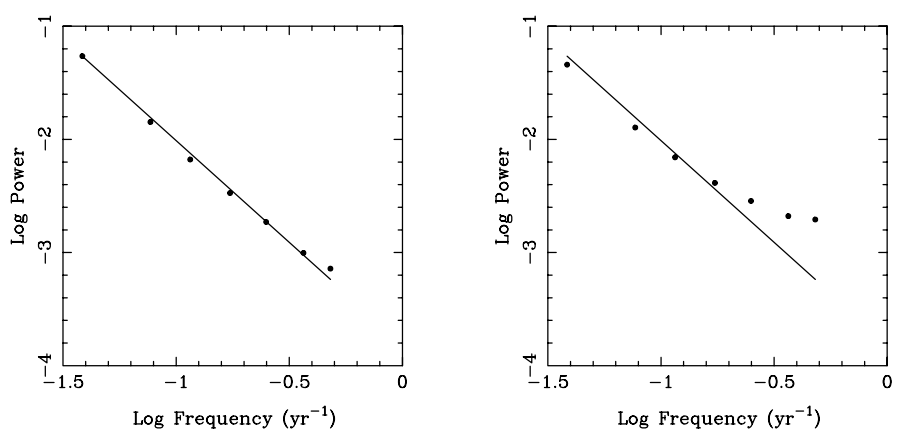

Fig. 3. Discrete Fourier power spectra for simulated light curves constructed from a power law power spectrum of index -1.8 , indicated by the solid line (left hand panel). The effect of adding Gaussian noise with standard deviation 0.06 mag is shown in the right hand panel.

the shape and features of a power spectrum are relatively easy to interpret. The windowing function due to short runs of data produces little distortion to the power spectrum, and Gaussian noise adds a constant term to the power spectrum, the size of which can be derived from the photometric errors and subtracted as a correction.

The effects described in the previous paragraph are illustrated in Fig. 3 which shows a Fourier power spectrum for simulated light curves constructed as described in the previous subsection. The solid line indicates the power law power spectrum used for the simulations, in this case with an index of -1.8 . The power spectrum of the 814 light curves of 26 epochs are shown as filled circles in the left hand panel of Fig. 3. It will be seen that very little deviation from the original power spectrum has been produced by the windowing process. The right hand panel shows the effect of adding Gaussian noise with a standard deviation of $0.06 \mathrm{mag}$ to the light curves. The effect on the power spectrum is to make a relative increase in the power of the short timescale frequencies, which can be corrected for by subtracting a constant from all frequencies, as described in the previous paragraph.

\section{The timescale of quasar variation}

In the previous section we have discussed the relative merits of structure function and Fourier power spectrum analysis, and there seems little doubt that for the purpose of analysing the data presented here to measure timescales, the latter approach is preferable. Accordingly, for the remainder of this paper we shall confine attention to Fourier power spectra of the data.

In the measurement of timescales in the AGN light curves, we shall consider two situations. Firstly we shall analyse Fourier power spectra for light curves as measured, that is in the observer's frame where no correction is made for effects of time dilation. Astrophysically, this corresponds to a situation where the variations do not originate in the quasar itself, but along the line of sight at much lower redshift. In particular, if the variations are caused by the microlensing effect of a cosmologically distributed population of compact bodies (Hawkins 1993, 1996), the most probable redshift for lensing peaks strongly at $z \approx 0.5$ (Turner et al. 1984), with only weak dependence on the redshift of the source. Thus the effects of time dilation will be small, and the observer's rest frame will be the most appropriate for measuring timescales. Secondly we shall carry out the analysis in the rest frame of the AGN, which is appropriate for intrinsic variations, and will necessitate a correction to remove the
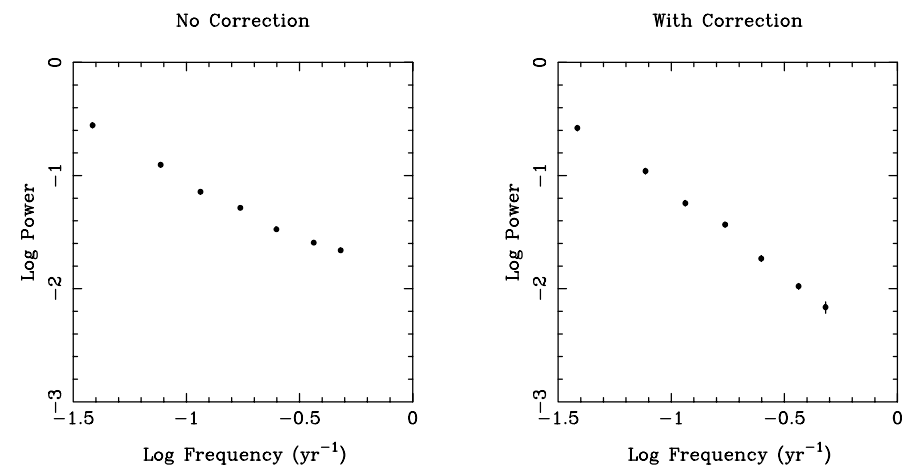

Fig. 4. Fourier power spectra for the sample of 814 quasars in Field 287 for which the structure function is shown in Fig. 1. The left hand panel shows the power spectrum as measured, and the right hand panel after correcting for measurement noise, as described in the text.

effects of time dilation to be applied to each light curve before the timescale is measured.

In fact, AGN are known to vary at least at some level both intrinsically and due to microlensing. Studies of low redshift quasars where there is no question of microlensing show variations in brightness at least at some level (Hawkins 2004), while gravitationally lensed systems such as the double quasar Q0957+561 show unambiguous evidence for microlensing (Pelt et al. 1998).

\subsection{Timescale in the observer's frame}

Figure 4 (left hand panel) shows the Fourier power spectrum for blue passband light curves for the sample of 814 quasars in Field 287, described above. For each quasar 26 epochs of observation are available, as described in Sect. 2. The Fourier power spectrum for the sample of light curves was evaluated as described above for the simulated data in the previous section. The error bars are derived from the dispersion of the individual power spectra. The shape of the power spectrum appears close to that of a power law, that is linear in the $\log / \log$ plot, but with a noticeable decrease in slope towards high frequencies.

In order to provide an accurate measurement of the true shape of the power spectrum, it was necessary to correct for the effects of measurement noise. This was done as described at the end of the previous section based on the measurement errors derived in Sect. 2. As a further check, the correction was obtained by measuring the power spectrum of a sample of stars assumed to be non-variable, thus providing a direct empirical measure of the measurement noise. These two approaches to error estimation gave similar results, and the right hand panel of Fig. 4 shows the result of making this correction, which basically has the effect of straightening and steepening the linear form of the power spectrum.

The power law increase in the power spectrum in Fig. 4 implies no preferred or characteristic timescale for the quasar variations. Detailed examination of the long timescale end does in fact suggest the possibility of a break in the slope, but given the relatively short baseline in frequency it is not possible to establish its significance in a formal way. The monitoring programme has already been running for nearly thirty years, and given the logarithmic nature of a power law relation, the amount of extra time needed to make a significant improvement in the frequency baseline is comparable to a human lifetime. An alternative approach is to add in shorter timescale measurements to provide a firmer measurement of the asymptotic slope in the log/log plot 
Combined Samples

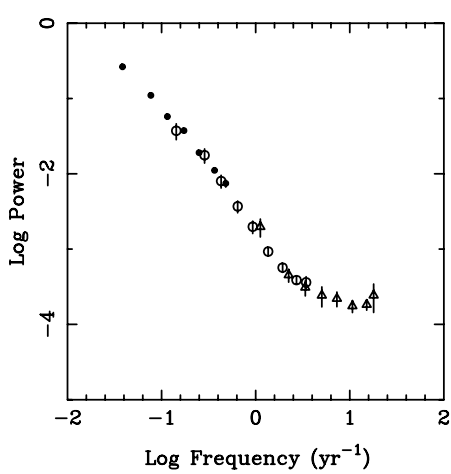

Fig. 5. Fourier power spectra of blue passband light curves in the observers frame for the sample of quasars in Field 287 sampled yearly (filled circles), the MACHO quasars with 50 day sampling (open circles) and 10 day sampling (open triangles).

towards high frequencies. The MACHO observations described in Sect. 2 provide an excellent if somewhat small database for this purpose.

Figure 5 shows Fourier power spectra for the combined Field 287 and MACHO data. The data from the right hand panel of Fig. 4 are shown as filled circles. The open circles show the power spectrum for the MACHO data with 50 day sampling interval, constructed as for the Field 287 sample. The correction for the windowing effect obtained from the errors in the MACHO data was applied to the to the 50-day sampling power spectrum as discussed above, although it was found to be small due to the relatively small errors in the CCD data. The situation was rather different for the 10-day sampled data. Here for the shorter timescales the random errors were comparable in size to the signal, and the correction could not be made with any confidence. The data was therefore left unchanged and modelled in the fitting procedure described below. There was no arbitrary normalisation applied to any of the power spectra, and the fact that in the area of overlap they are coincident is solely attributable to the homogeneity of the data and reduction procedures. The third set of data in Fig. 5, shown as open triangles, is from the same set of MACHO observations but with 10 day sampling. The Fourier power spectrum is produced in the same way as for the data with 50 day spacing. Inspection of Fig. 5 shows that all three sets of data points consistently map out the same curve, both in the sense of continuity of slope, and agreement in areas of overlap. This is perhaps not too surprising for the two different samplings of the MACHO data, but the very good agreement between these and the long term data from Field 287 implies that sample selection effects are not significantly distorting the underlying spectrum of variability.

The overall shape of the function in Fig. 5 comprises a linear section in the middle, which flattens to a constant at frequencies corresponding to timescales of less than about 30 days, which appears to be the effect of measurement noise from the 10 day sample for which no correction was made. Towards low frequencies the power spectrum appears to maintain a linear (power law) relation, with only a slight indication of a turn over. In order to quantify a possible change of slope, and hence an associated timescale, we use the function

$P(f)=\frac{C}{\left(1+\frac{f}{f_{\mathrm{c}}}\right)^{a}}+d$.
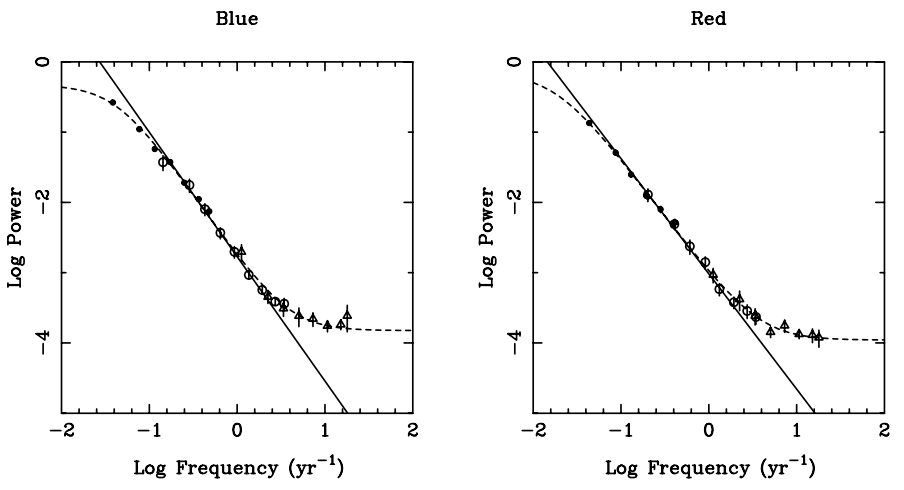

Fig. 6. Fourier power spectra of blue passband light curves (left hand panel) and red passband light curves (right hand panel) in the observer's frame for quasars in the Field 287 and MACHO samples. Symbols are as for Fig. 5. The dashed line is the best-fit curve of Eq. (3), and the solid line is the asymptotic power law relation for $P(f)$ as described in the text.

This is a small modification of a function employed for the purpose of measuring timescales of X-ray variabilty (Edelson \& Nandra 1999). The function describes the power spectrum $P(f)$ of a power law relation with index $a$ at high frequencies which breaks to a constant value $c$ at a cut-off frequency $f_{\mathrm{c}}$. The modification is to add a constant term $d$ to model the flattening attributed to noise at high frequencies. The function $P(f)$ was fitted iteratively to the data by varying the four parameters $a, f$, $c$ and $d$ and looking for a minimum $\chi^{2}$, which was measured as 28.3 with 23 degrees of freedom. The best fit for $P(f)$ is shown as a dashed line in the left hand panel of Fig. 6. Also shown, as a solid line, is the asymptotic power law relation for $P(f)$. This has index $a$, with best-fit value $a=-1.77$. The "cut-off" frequency $f_{\mathrm{c}}$, which is of central importance to this investigation, has a best fit value corresponding in the time domain to $24.0 \pm 2.7$ years. Given that the total run of data is only 25 years, this figure should be treated as a lower limit to the timescale of variation. The error estimates were obtained by selecting a large number of sub-samples of the data and carrying out the same parameter estimation procedure as for the full sample. The rms dispersion in the measurement of the cut-off frequency $f_{\mathrm{c}}$ was then taken as the error.

In addition to the light curves in the $B_{J}$ passband, light curves were also available in $R$. These observations, described in Sect. 2, are somewhat inferior to the $B_{J}$ observations in several respects. Firstly, they were only started in 1980, and so comprise 23 epochs as opposed to 26 . Secondly, due to the blue colour of most of the quasars, a significant number were not included in the $R$ sample because they were not detected in the $R$ band in one or more epochs. This resulted in a sample of 403 quasars with complete $R$ band light curves compared with 814 for the $B_{J}$ band. Another problem arising from the blue colour of most quasars is that the $R$ band measures tend to be closer to the the plate limit, and hence noisier. Finally, the smaller amplitude of many AGN in the $R$ band (Hawkins 2003) means that there is less power in the Fourier power spectrum, and hence poorer signal-to-noise in the detection of any features. Despite these limitations, we have analysed the red passband light curves as for the blue, and show the results in the right hand panel of Fig. 6. The best fit value for the cut-off frequency in the time domain is $70.9 \pm 33.4$ years. Given that the data only cover 22 years, we shall take that as a lower limit for the timescale of variation. 
Blue

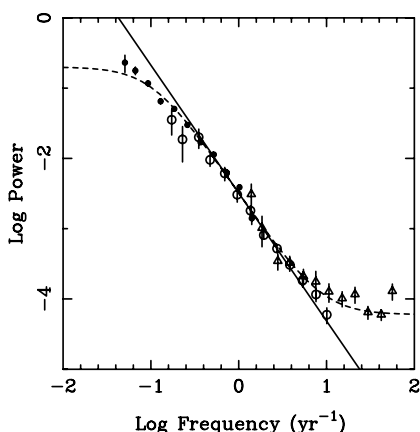

Red

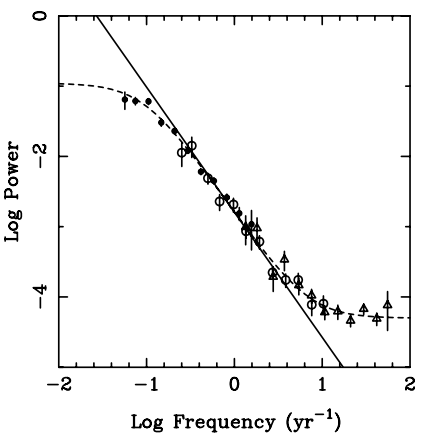

Fig. 7. Fourier power spectra in the quasar rest frame for the data in Fig. 6. The best fit curves to the data are also shown as for Fig. 6.

\subsection{Timescale in the quasar rest frame}

The data in Fig. 6 are all in the observer's rest frame, with no correction for any effect of time dilation, as discussed at the beginning of this section. However, if the observed variation is intrinsic to the quasars at cosmological distances, one would expect a time dilation effect, and for the effect to be large. In order to allow for time dilation and reduce the observations to the rest frame of the quasar, it is necessary to change the timescale of variation of each individual quasar of redshift $z$ by a factor $(1+z)^{-1}$, and hence the frequency scale by a factor $(1+z)$. This leads to a modification of Eq. (2) such that

$\tau \rightarrow \frac{\tau}{1+z}$.

The two panels of Fig. 7 each show three Fourier power spectra constructed from the same blue and red passband light curves for Fig. 6, but with the modifications to Eq. (2) given in Eq. (4). The expected effect of extending the power spectrum to higher temporal frequencies is clearly visible, and although an approximate power law shape is retained the shape at low frequencies now shows a noticeable departure from a power law (linear) relationship. In order to measure timescales, the same procedure was carried out as for the data in Fig. 6, involving fitting the function in Eq. (3). The best fit values for the blue and red passbands were $9.7 \pm 0.1$ and $11.4 \pm 0.6$ years respectively. These values are in good agreement, and well below the timespan of the data giving confidence that a real timescale is being measured.

\section{Accretion disc structure}

Until recently the size of AGN accretion discs was not known with any degree of certainty. We first note that the accretion radius $r_{\text {acc }}$ given by

$r_{\mathrm{acc}}=\frac{2 G M}{c_{\infty}^{2}}$

where $c_{\infty}$ is the sound speed at infinity is an absolute upper limit for the size of an accretion disc around a black hole of mass $M$. For a quasar this is a huge distance, around 30 parsecs, and not restrictive as an upper limit. A much more stringent limit is derived by Goodman (2003), who uses the Toomre instability parameter for Keplerian motion

$Q \approx \frac{\Omega^{2}}{2 \pi G \rho}$

to derive a radius $r_{Q=1}$ beyond which $Q<1$ and local gravitational instability occurs, and hence self-gravity terminates the disc structure. For typical parameter values this occurs at $r_{Q=1} \sim 10^{-2} \mathrm{pc}$. Goodman then goes on to examine various ways of creating larger quasar discs, but concludes that they probably do not extend beyond $10^{-2}$ pc or 1000 Schwarzschild radii.

An alternative approach to determining the size of accretion discs is discussed by Lyubarskii (1997). In this paper he makes the point that fluctuations in the accretion rate lead to flicker noise, and a power law Fourier power spectrum, with a cut-off related to the radius of the outer edge of the accretion disc. In his model, long timescale fluctuations are generated in the outer part of the accretion disc and propagate inwards where they cause changes in brightness in the optically emitting region near the disc centre. The outer radius $r_{\text {out }}$ of the accretion disc is given by Lyubarskii as

$r_{\text {out }}=\left(\alpha \sqrt{G M} f_{\min }^{-1}\right)^{2 / 3}$

where $\alpha$ is the viscosity parameter and $f_{\min }$ is the frequency of the break in the power law spectrum of variations. King et al. (2004) develop this scheme further with an explicit physical model for disc variability, invoking local dynamo processes driving angular momentum loss through an outflow wind.

The frequency $f_{\min }$ in Eq. (7), as defined by Lyubarskii (1997), is basically the same frequency $f_{c}$ from Eq. (3) measured as the break in the power law in Fig. 7. If we substitute the rest frame value from above of about 11 years for $f_{\min }^{-1}$ in Eq. (7), and adopt $\alpha=0.1$ (Balbus \& Hawley 1998; Goodman 2003) we obtain $r_{\text {out }}=10^{-2}$ pc or $3 \times 10^{16} \mathrm{~cm}$.

\section{Microlens masses}

In the event that the quasar variations are dominated by the effects of microlensing, it is appropriate to use the light curves measured in the observer's frame, and the cut-off frequency $f_{\mathrm{c}}$ from Eq. (3) is a measure of the characteristic mass of the microlenses. To see this we note that the distance over which the gravitational field of a lensing object has a significant effect on the image, known as the Einstein radius $\theta_{\mathrm{e}}$, and defined by

$\theta_{\mathrm{e}}=\left\{\frac{4 G m}{c^{2} R}\right\}^{\frac{1}{2}}$

varies as the square root of the lens mass $m$. In this equation $R$ is the distance to the lens and $G$ and $c$ are the gravitational constant and speed of light respectively. If the lensing object is moving across the line of sight with tangential velocity $v_{\mathrm{t}}$, the image will brighten and fade on the timescale that the source lies within the Einstein radius. Hence the characteristic timescale of variation $t_{\mathrm{c}}$ will be given by

$t_{\mathrm{c}}=\frac{R \theta_{\mathrm{e}}}{v_{\mathrm{t}}}$

and combining Eqs. (8) and (9)

$m=\frac{c^{2}}{2 G} \frac{v_{\mathrm{t}}^{2} t_{\mathrm{c}}^{2}}{R}$

Thus $m$ varies as $t_{\mathrm{c}}^{2}$. If we adopt a characteristic value of $600 \mathrm{~km} \mathrm{~s}^{-1}$ for $v_{\mathrm{t}}$ and assume that most lenses lie at a redshift $z \sim 0.5$ (Turner et al. 1984) then by using the lower limit to the timescale of 24 years measured from the data in Fig. 6, we can obtain a value for the minimum microlens mass $m \sim 0.4 M_{\odot}$. 


\section{Discussion}

After the first primitive estimates of timescale discussed in the Introduction, and when the Fourier power spectrum was plotted in the form of a $\log / \log$ plot, it was clear that there was no obvious break in a linear relation. This situation persisted as more data was added, and it was in this context that the short term MACHO data was added as the only realistic way of extending the baseline. It is quite remarkable that for the case of the observer's reference frame the straight line relationship was maintained, covering three orders of magnitude in time and reinforcing the result of Hawkins (2001).

Until recently, little attention had been paid in this project to an analysis of the light curves in the quasar rest frame. This was largely due to the author's interest in the microlensing interpretation of quasar variability where the observer's frame is more appropriate. It came therefore as something of a surprise to find a clearly measurable break in the power spectrum for data analysed in the quasar frame. This meant that a definite measure of timescale could be made rather than a lower limit, and has interesting implications for quasar accretion discs.

The figure for the outer radius of the accretion disc of $3 \times 10^{16} \mathrm{~cm}$ refers to the entire disc out to the point where graviational instability sets in. It is interesting to compare this with measurements of disc size from microlensing in multiply lensed quasar systems. In this situation the radius which is measured is that of the luminous part of the disc. For the system Q0957+561, Pelt et al. (1998) find for the source radius $r_{\mathrm{s}} \sim 3 \times 10^{15} \mathrm{~cm}$, and for Q2237+0305 Kochanek (2004) also finds $r_{\mathrm{s}} \sim 3 \times 10^{15} \mathrm{~cm}$. The optically luminous part of the disc would thus appear to be about a tenth of the diameter of the whole disc.

If we assume that the observed variations are intrinsic to the quasar then the coincidence between $r_{Q=1}$ and $r_{\text {out }}$ is remarkable, given the completely different bases on which they were calculated. It is interesting to note that $10^{-2} \mathrm{pc}$ or 10 light days is also the canonical value for the size of the broad line region in AGN (Peterson et al. 1999). It is tempting to equate the break up of the outer edge of the accretion disc due to gravitational instability with the cloud structure and turbulence of the broad line region. Should this measurement of the size of the accretion disc prove robust, it will provide a fundamental constraint on the structure of the unified model, and facilitate the determination of other model parameters. However, before this measurement can be accepted with confidence, there are still some issues which need to be addressed.

The small size of the accretion disc presents some difficulties, which have been discussed by Goodman (2003) in relation to his own work. The main problem is to find a way of replenishing the disc with gas of small angular momentum at a high enough rate to build a super-massive black hole. His suggestion that it might come from a low angular momentum tail of gas from the bulge seems quite plausible.

To make full use of the results reported here it is most important that Fourier power spectra of synthetic light curves for various accretion disc models are made available in the literature. Typically, power spectra take the form of a power law rise to low frequencies, terminated by a break corresponding to some characteristic timescale. Free parameters include the power law index which reflects the nature of the accretion process, the break frequency which is a measure of the size of the accretion disc, and the amplitude which depends on the mass diffusion rate. A good start towards producing testable models has already been made (Kawaguchi et al. 1998; King et al. 2003), and hopefully there is more to come.
It is interesting to note that the power law index measured for optical variations in this paper $(\alpha=-1.77)$ is similar to that found for X-ray variations. For example, Edelson \& Nandra (1999) find $\alpha=-1.74$ for NGC 3516, and Uttley et al. (2002) obtain similar results for a small sample of AGN. However, the timescales vary by two orders of magnitude between optical and $\mathrm{X}$-ray, and it is clear that different things are being measured in the two passbands. Understanding the relationship between the two provides an interesting challenge.

In the event that the observed variation is dominated by the effects of microlensing, the lower limit of $m \sim 0.4 M_{\odot}$ for the microlensing bodies is considerably larger than earlier estimates using this approach (Hawkins 1996). Such objects would lie in the same mass range as those detected by the MACHO project (Alcock et al. 1997), although they would have to be more abundant to be responsible for the extent of variability observed. Estimates of lens mass from the study of microlensing events in multiply lensed quasar systems tend to give upper limits somewhat similar to the lower limit quoted above for the present work. For example, Kochanek (2004) finds an upper limit to the mean of the mass function of microlensing bodies in the system Q2237+0305 to be around $m \leq 0.2 M_{\odot}$, and Refsdal et al. (2000) find an upper limit to the mass of microlensing bodies in the quasar system Q0957+561 of $m \leq 0.5 M_{\odot}$.

\section{Conclusions}

In this paper we have combined datasets from two AGN monitoring programmes to construct Fourier power spectra in blue and red passbands on timescales from 10 days to 25 years. We have investigated different methods of measuring timescales of variation in AGN light curves, and concluded that Fourier power spectrum analysis provides the best approach. We have used the combined datasets to construct Fourier power spectra covering nearly three orders of magnitude in time in both the observer's frame and the quasar rest frame. Timescales were then measured by fitting a function designed to detect a turn over in the power spectrum.

If the variations are interpreted as resulting from gravitational microlensing then it is appropriate to use the observer's frame to measure timescales. In the observer's frame the power spectrum was found to have an approximately power law shape with no significant break, and a lower limit of 24 years was obtained for the timescale of variation. This corresponds to a minimum mass for microlensing bodies of $m \sim 0.4 M_{\odot}$. This mass is larger than that found in earlier studies of quasar variation, and typical of the mass of bodies found in the Galactic halo by the MACHO project.

If the variations are seen as intrinsic to the AGN then the effect of time dilation must be corrected for. This results in a modified Fourier power spectrum which retains its power law shape on short timescales, but with a break towards higher frequencies. This break was shown to correspond to 11 years in the quasar rest frame. We interpret this as a characteristic timescale for variations intrinsic to the AGN. This timescale is used to estimate the size of an AGN accretion disc which we find to be $10^{-2} \mathrm{pc}$ or about 10 light days. This is consistent with the radius at which the onset of self-gravity may terminate the disc, and roughly coincident with the position of the broad line region. This suggests the possibility that the broad line region is associated with the break up of the outer part of the accretion disc. 


\section{References}

Alcock, C., Allsman, R. A., Alves, D., et al. 1997, ApJ, 486, 697 Balbus, S. A., \& Hawley, J. F. 1998, Rev. Mod. Phys., 70, 1

Cristiani, S., Trentini, S., La Franca, F., et al. 1996, A\&A, 306, 395

de Vries, W. H., Becker, R. H., \& White, R. L. 2003, AJ, 126, 1217

de Vries, W. H., Becker, R. H., White, R. L., \& Loomis, C. 2005, AJ, 129, 615

Edelson, R., \& Nandra, K. 1999, ApJ, 514, 682

Geha, M., Alcock, C., Allsman, R. A., et al. 2003, AJ, 125, 1

Goodman, J. 2003, MNRAS, 339, 937

Hawkins, M. R. S. 1993, Nature, 366, 242

Hawkins, M. R. S. 1996, MNRAS, 278, 787

Hawkins, M. R. S. 2000, A\&AS, 143, 465

Hawkins, M. R. S. 2001, ApJ, 553, L97

Hawkins, M. R. S. 2002, MNRAS, 329, 76

Hawkins, M. R. S. 2003, MNRAS, 344, 492

Hawkins M. R. S. 2004, A\&A, 424, 519

Hook, I. M., McMahon, R. G., Boyle, B. J., \& Irwin, M. J. 1994, MNRAS, 268, 305
Kawaguchi, T., Mineshige, S., Umemura, M., \& Turner, E. L. 1998, ApJ, 504, 671

King, A. R., Pringle, J. E., West, R. G., \& Livio, M. 2004, MNRAS, 348, 111

Klimek, E. S., Gaskel, C. M., \& Hedrick, C. H. 2004, ApJ, 609, 69

Kochanek, C. S. 2004, ApJ, 605, 58

Lyubarskii, Yu. E. 1997, MNRAS, 292, 679

Pelt, J., Schild, R., Refsdal, S., \& Stabell, R. 1998, A\&A, 336, 829

Peterson, B. M., Barth, A. J., Berlind, P., et al. 1999, ApJ, 510, 659

Refsdal, S., Stabell, R., Pelt, J., \& Schild, R. 2000, A\&A, 360, 10

Schneider, D. P., Fan, X., Hall, P. B., et al. 2003, AJ, 126, 2579

Sesar, B., Svilkovic, D., Ivezic, Z., et al. 2006, AJ, 131, 2801

Stalin, C. S., Gopal-Krishna, S. R., \& Wiita, P. J. 2004, MNRAS, 350, 175

Trevese, D., Kron, R. G., Majewski, S. R., \& Bershady, M. A. 1994, ApJ, 433, 494

Turner, E. L., Ostriker, J. P., \& Gott, J. R. 1984, ApJ, 284, 1

Uttley, P., McHardy, I. M., \& Papadakis, I. E. 2002, MNRAS, 332, 231

Vanden Berk, D. E., Wilhite, B. C., Kron, R. G., et al. 2004, ApJ, 601, 692 